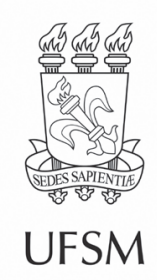

\title{
Artigos
}

\section{Estrutura de uma Floresta Ombrófila Densa Montana com dominância de Euterpe edulis Mart.}

\author{
Structure of a Dense Montana Ombrophilous Forest with dominance of \\ Euterpe edulis Mart.
}

\author{
Letícia Bonifácio Fávaro' ${ }^{\oplus}$ \\ Agostinho Lopes de Souzall $\odot$ \\ Renato Totti Maia"II $\odot$ \\ Ricardo de Oliveira Gaspariv $\odot$ \\ 'Pesquisadora Autônoma, Botuverá, SC, Brasil \\ "Universidade Federal de Viçosa, Viçosa, MG, Brasil \\ '"Instituto do Meio Ambiente de Santa Catarina, Botuverá, SC, Brasil \\ "Vniversidade Federal de Brasília, Brasília, DF, Brasil
}

\section{RESUMO}

O presente trabalho analisou as estruturas horizontal, vertical, interna e paramétrica de uma Floresta Ombrófila Densa Montana, com dominância da espécie Euterpe edulis Mart. Foram inventariadas, aleatoriamente, 20 parcelas de $10 \mathrm{~m} \times 50 \mathrm{~m}$, totalizando 1,0 ha de área amostral. $\mathrm{O}$ critério de inclusão foi o diâmetro do tronco, medido a 1,30 m do solo (DAP) igual ou superior a 5,0 cm, que resultou em 2.394 indivíduos.ha-1, 2.594 fustes.ha-1 ${ }^{-1}$ dominância total de $42,20 \mathrm{~m}^{2}$.ha-1, sendo que 7,81 $\mathrm{m}^{2}$.ha-1 corresponderam a 642 indivíduos.ha-1 de Euterpe edulis. Os diâmetros médios foram de 12,33 cm e de $11,48 \mathrm{~cm}$ para a comunidade e para a população de Euterpe edulis, respectivamente. No estrato médio da floresta, definido entre 5 a 12 metros de altura total, encontram-se $65 \%$ dos indivíduos de Euterpe edulis. A estrutura diamétrica da comunidade florestal apresentou distribuição em forma de "J-invertido", mas desbalanceada. No tocante à estrutura interna, 96,4\% dos indivíduos apresentaram epífitas, 57,8\% com ausência de trepadeiras e 62,5\% dos indivíduos com ausência de cipós. Essas características, associadas ao efeito edafoclimático da área de estudo e à ausência do fenômeno de caducifoliedade, permitiram classificar o fragmento em Floresta Ombrófila Densa Montana em estágio médio avançado de regeneração. Quanto à população de Euterpe edulis, a representação da espécie pela área basal demonstrou sua dominância na comunidade florestal, sendo observada uma população madura, apesar da subestimação dos indivíduos adultos.

Palavras-chave: Estágio sucessional; Parâmetros estruturais; Palmeira-juçara 


\section{ABSTRACT}

The current article analyzed the horizontal, vertical, internal and parametric structures of a Dense Montana Ombrophilous Forest with a dominance of Euterpe edulis Mart. species. Twenty (20) sections of $10 \times 50$ m were inventoried contingently, totaling 1.0 ha of sampling area. The criteria for inclusion was the diameter at the breast height, measured $1.30 \mathrm{~m}$ above ground level (DBH), equal or greater than 5.0 $\mathrm{cm}$, which resulted in 2,394 indiviuals.ha-1; 2,594 boles.ha-1, total dominance of $42.20 \mathrm{~m}^{2}$. ha-1, wherein $7.81 \mathrm{~m}^{2}$.ha-1 corresponded to 642 individuals. ha-1 of Euterpe edulis. The average diameters were 12.33 $\mathrm{cm}$ and $11.48 \mathrm{~cm}$ for the community and the population of Euterpe edulis, respectively. The medium extract of the forest, defined at a height of 5 to $12 \mathrm{~m}$ total size revealed $65 \%$ Euterpe edulis individuals. The diametric structure of the forest community showed distribution in the form of "inverted-J", but unbalanced. As for the internal structure, $96.4 \%$ of the individuals showed epiphytes, $57.8 \%$ without creepers, and $62.5 \%$ of individuals without lianas. These characteristics associated to the edaphoclimatic effect of the studied area and absence of a deciduousness phenomenon, permitted classifying the fragment as Dense Montana Ombrophilous Forest in an intermediated-advanced stage of regeneration. As for the Euterpe edulis population, the species representation by the basal area demonstrated its dominance in the forest community, showing a mature population in spite of underestimated adult individuals.

Keywords: Successional stage; Structural parameters; Juçara palm

\section{INTRODUÇÃO}

Tal qual a maioria das áreas de domínio da Mata Atlântica, a Zona da Mata de Minas Gerais sofre com intensa pressão antrópica que causa, dentre outros impactos, redução e degradação da cobertura vegetal original. Atualmente, nesta região predominam fragmentos florestais isolados (TABARELI et al., 2005; RIBEIRO et al., 2009b; FUNDAÇÃO SOS MATA ATLÂNTICA; INSTITUTO NACIONAL DE PESQUISAS ESPACIAIS, 2015), cuja tipologia varia entre Floresta Ombrófila Densa (RIBEIRO, 2003; OLIVEIRA-FILHO, 2006) a Floresta Estacional Semidecidual (VELOSO; RANGEL FILHO; LIMA, 1991; OLIVEIRA-FILHO, 2006; INSTITUTO ESTADUAL DE FLORESTAS, 2008), sendo que alguns destes fragmentos ocorrem em elevadas altitudes (RIBEIRO, 2003; SAPORETTI JÚNIOR, 2005).

Pertencente à família Arecaceae, a palmeira-juçara (Euterpe edulis Mart.) ocupa o estrato médio da Floresta Ombrófila Densa, ocorrendo desde o sul da Bahia até o norte do Rio Grande do Sul, com distribuição preferencial ao longo do litoral brasileiro, 
no domínio da Floresta Ombrófila Densa, ocorrendo também na maior parte das formações Estacional Decidual e Semidecidual (CARVALHO, 2003; HIGASHIKAWA, 2009). Apesar da ampla distribuição geográfica, poucos são os trabalhos que citavam Minas Gerais como área de ocorrência da palmeira-juçara (REITZ, 1974; CARVALHO, 1994), embora seja encontrada em diversos estudos realizados em Minas Gerais (IRSIGLER, 2002; CAMPOS et al., 2006; HIGUCHI, 2006; LEMOS, 2008; SILVA JÚNIOR, 2008; HIGASHIKAWA, 2009; RIBEIRO et al., 2009a; LORENZON, 2011).

No entanto, devido à extração do palmito e à fragmentação florestal, esta palmeira sofreu drástica redução, chegando a desaparecer em algumas regiões de ocorrência. Atualmente, está inclusa na Lista Oficial das Espécies da Flora Brasileira Ameaçada de Extinção (BRASIL, 2014) na categoria vulnerável.

Diante disso, o objetivo deste trabalho foi classificar o estágio sucessional da comunidade, mediante aplicação da legislação vigente, caracterizar a distribuição e analisar a estrutura de uma população de Euterpe edulis ocorrendo em Floresta Ombrófila Densa de Altitude na região da Zona da Mata de Minas Gerais, com foco no potencial de produção de frutos subsidiando projetos de manejo de produto florestal não madeireiro.

\section{MATERIAIS E MÉTODOS}

A área de estudo (Figura 1), com centroide nas coordenadas geográficas $20^{\circ} 54^{\prime} \mathrm{S}$ e $42^{\circ} 32^{\prime} \mathrm{W}$ e altitudes variando de $1.010 \mathrm{~m}$ a $1.380 \mathrm{~m}$, é um fragmento de Floresta Ombrófila Densa Montana pertencente ao bioma Mata Atlântica localizado no município de Muriaé - MG, a menos de 2 km de distância do limítrofe sul do Parque Estadual da Serra do Brigadeiro. Os solos predominantes são Latossolo VermelhoAmarelo e Latossolo Vermelho-Amarelo Húmico, Cambissolos e Litossolos (ENGEVIX, 1995). O clima, segundo a classificação de Köppen, é Cwb (IBGE, 2012; SÁ JúNIOR et al., 2012; ÁLVARES et al., 2013). A temperatura média anual é de aproximadamente $18^{\circ} \mathrm{C}$, com os meses mais quentes apresentando uma média inferior a $23^{\circ} \mathrm{C}$ e uma 
média inferior a $17^{\circ} \mathrm{C}$ nos meses mais frios (MOREIRA, 2008). A umidade relativa do ar se mantém em torno de $80 \%$ até mesmo no período mais seco (MOREIRA, 2008). A precipitação média anual é de aproximadamente $1.500 \mathrm{~mm}$, com período seco entre os meses de abril a outubro (RAMOS; SANTOS; FORTES, 2009).

Figura 1 - Visualização da área de estudo, com croqui representativo dos indivíduos

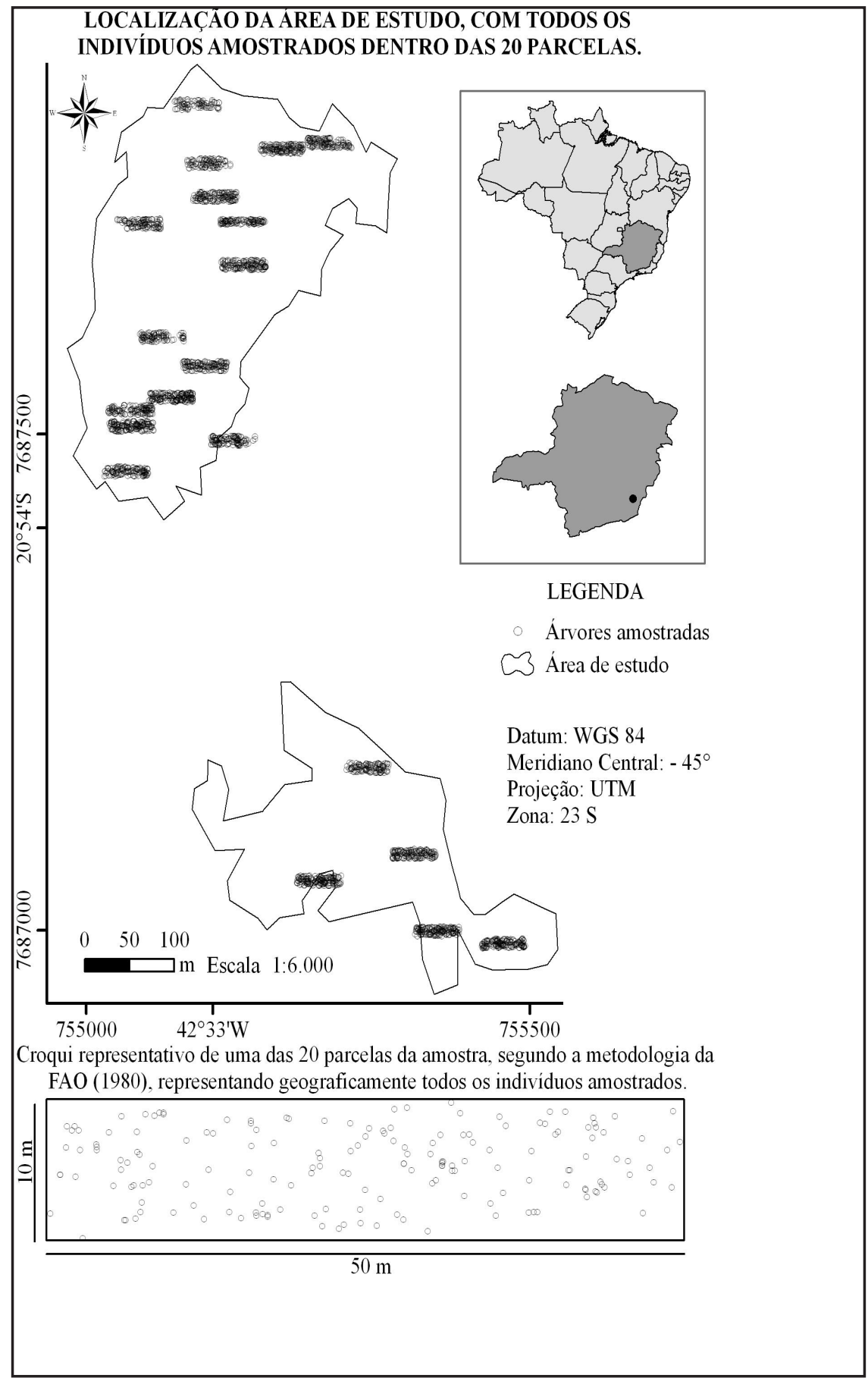

Fonte: Autores (2020) 
Compondo um total de 13,5 ha, as duas subáreas foram inventariadas por meio da instalação, aleatória, de 20 parcelas permanentes medindo 10 m x 50 m (500 m²) cada uma, totalizando 1,0 ha de área amostral. Para cada indivíduo arbóreo (DAP $\geq$ 5,0 cm) foi medido o CAP, estimada a altura total $(\mathrm{Ht})$ e localizado suas coordenadas cartesianas $(\mathrm{x}, \mathrm{y})$ de acordo com Food and Agriculture Organization of the United Nations (1980). Para esses indivíduos também foram registradas as ocorrências de epífitas (atribuindo-se um código quando se tratava de liquens, briófitas e/ou pteridófitas, e outro para orquídeas, bromélias e/ou cactos), trepadeiras (diferenciadas entre herbáceas e lenhosas), cipós no tronco, na copa ou em ambos, qualidade de copa e iluminação de copa.

Para caracterizar a estrutura da floresta nativa, considerou-se o número de indivíduos e de fustes para todas as espécies amostradas e separadamente para Euterpe edulis, para a população total e por parcela, sendo analisados parâmetros das estruturas horizontal, vertical, interna e diamétrica. Os indivíduos mortos não foram incluídos nas análises.

De posse dos números de indivíduos por parcela, estimou-se, por meio do Índice de Morisita (BROWER; ZAR, 1984; KREBS, 1999), o padrão de distribuição espacial de Euterpe edulis na comunidade.

Para definir estágio de sucessão de Mata Atlântica em Minas Gerais, classificouse e interpretou-se a frequência e predominância (\%) dos fustes por parcela nas classes de altura total (Ht) e de diâmetro médio (Dg), conforme descrito na Resolução Conama n. 392 (BRASIL, 2007). Ainda, as alturas totais dos fustes, por espécie, foram distribuídas em classes de altura com amplitudes de 1 metro e 2 metros, a partir da altura mínima de 1,3 m e ajustadas funções de densidade probabilística (MACHADO et al., 2010).

Para analisar a distribuição diamétrica da comunidade florestal foram considerados todos os fustes amostrados com DAP $\geq 5 \mathrm{~cm}$ e amplitudes ou intervalos de classe de diâmetro de 1,0 cm, 2,0 cm e 5,0 cm. De posse desses dados, foram ajustadas funções de densidade probabilística (f.d.p), visto que essas permitem obter 
a probabilidade de as árvores ocorrerem dentro de determinados intervalos de classes de diâmetros e, ou, de alturas (MACHADO et al., 2010). Para verificar se as distribuições diamétricas são balanceadas, calculou-se o quociente (" $q$ ") de De Liocourt (MEYER, 1952), dividindo-se sucessivamente o número de indivíduos por hectare da j-ésima classe diamétrica (DAj) pelo número de indivíduos por hectare da classe diamétrica seguinte $\left(D A_{j+1}\right)$, ou seja, $q_{j}=D A_{j} / D A_{j+1}$.

Também foram analisados, para a população de Euterpe edulis, os números de indivíduos.ha-1 nas diferentes fenofases (floração ou inflorescências, frutificação ou infrutescências, ou sementes). Esse critério foi determinante para analisar o potencial de produção de frutos da população natural de Euterpe edulis.

Para testar o ajuste de funções de densidade de probabilidade e outras estatísticas utilizou-se o teste de qui-quadrado (GOMES, 1987) e o teste t (LEITE; OLIVEIRA, 2002), a 5\% de significância.

\section{RESULTADOS E DISCUSSÃO}

$\mathrm{Na}$ comunidade, considerando-se todas as espécies e fustes com DAP $\geq 5$ cm, foram amostrados 2.394 indivíduos. ha-1 $^{-1}$ e 2.594 fustes.ha $^{-1}$, cuja área basal ou dominância absoluta foi de $42,20 \mathrm{~m}^{2}$.ha-1 . A espécie Euterpe edulis contribuiu com $27 \%$ dos indivíduos amostrados (642 ind.ha-1 $)$, perfazendo 7,808 $\mathrm{m}^{2}$.ha-1, ou seja, DoR de aproximadamente $46 \%$. Os diâmetros médio e máximo para Euterpe edulis foram 11,48 $\mathrm{cm}(\mathrm{S}= \pm 4,91 \mathrm{~cm})$ e 23, $39 \mathrm{~cm}$, respectivamente. Examinando-se apenas os indivíduos de Euterpe edulis que apresentaram estipe exposta acima de 1,30 m de altura, foram encontradas densidades superiores a 500 indivíduos.ha-1 (VELOSO; KLEIN, 1957), a

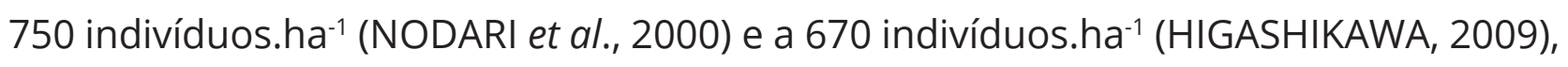
sendo considerada, por esses autores, uma das espécies de maior densidade dentro da Mata Atlântica, contribuindo para corroborar a dominância de Euterpe edulis na área de estudo.

Tanto a densidade populacional (DA e DR), quanto a dominância (DoA e DoR) e de Euterpe edulis comparativamente à comunidade, foram inferiores aos respectivos 
parâmetros de um fragmento de floresta paludosa (TEIXEIRA; ASSIS, 2005). Já para os demais estudos em fragmentos localizados em Minas Gerais com ocorrência de Euterpe edulis (IRSIGLER, 2002; SILVA, 2003; CAMPOS et al., 2006; PINTO, 2007) e em estudos realizados em outros estados com ocorrência de Mata Atlântica (REIS, 1995; BORÉM; OLIVEIRA-FILHO, 2002; MORENO; NASCIMENTO; KURTZ, 2003; SILVA et al., 2009; LIMA; CORDEIRO; MORENO, 2011; PRATA; ASSIS; JOLY, 2011), o presente trabalho apresentou os maiores valores de DA, DR, DoA e DoR.

O diâmetro da comunidade variou de 5,0 cm (diâmetro mínimo) a 72,96 cm (diâmetro máximo), com média aritmética dos diâmetros de $12,33 \mathrm{~cm}$ e diâmetro médio quadrático de 14,38 cm. Já a altura média aritmética da comunidade foi de $8,25 \mathrm{~m}$ com desvio padrão de \pm 4,01 m. De acordo com a Resolução Conama n. 392 (BRASIL, 2007), o diâmetro médio quadrático igual a $14,38 \mathrm{~cm}$ enquadra-se no estrato médio (10,0 $\mathrm{cm} \leq \mathrm{Dg}<20,0 \mathrm{~cm}$ ). Para a análise da estrutura vertical, foram considerados todos os fustes dos indivíduos com $D A P \geq 5,0 \mathrm{~cm}$. A estratificação feita com base na Resolução Conama n. 392 (BRASIL, 2007) permitiu definir o estágio de sucessão da comunidade florestal, considerando-se a frequência de ocorrência em cada um dos estratos (Tabela 1). A amostragem aleatória comtemplou satisfatoriamente a distribuição das parcelas, assim, optou-se por aplicar os critérios da referida resolução a cada parcela, tendo como resultado a classificação de trechos do fragmento que apresentam estrutura vertical variada, em que 65\% das parcelas apresentam estrutura de transição entre o estágio médio com forte componente de estágio avançado, de acordo com o critério de estratificação vertical (Tabela 1).

Verifica-se na Tabela 1 que a maioria das parcelas apresentou predomínio de indivíduos nos estratos médio e superior, que levou à interpretação do estágio de sucessão em uma transição da área de estudo. O dossel superior a 12 metros de altura abrangeu $18 \%$ dos indivíduos da comunidade florestal, podendo-se deduzir que a comunidade se encontra em estágio avançado de sucessão. Somente as parcelas 4, 17, 18, 19 e 20 representam ecounidades em estágio médio de sucessão, visto que não apresentam nítido dossel superior. As demais parcelas apresentam os três estratos, 
obviamente com predomínio de indivíduos no dossel médio (Tabela 1). Vale enfatizar que uma floresta nativa em estágio sucessional médio-avançado ou avançado-clímax precisa apresentar os três estágios bem definidos, ou seja, para a ocorrência de estágio médio é necessário que a comunidade tenha características de estágio inicial, como espécies indicadoras e estratos de diâmetro e altura. O mesmo vale para comunidades em estágio avançado de sucessão, ou seja, é fundamental a ocorrência de indicadores que caracterizem o estágio médio.

Tabela 1 - Definição do estágio sucessional da comunidade, por parcela, de acordo com a Resolução Conama n. 392

\begin{tabular}{|c|c|c|c|c|c|c|c|c|}
\hline \multirow{2}{*}{ Parcela } & \multicolumn{3}{|c|}{ Frequência (n.ha-1) } & \multirow{2}{*}{ (n.ha-1) } & \multicolumn{3}{|c|}{ Porcentagem (\%) } & \multirow{2}{*}{$\begin{array}{c}\text { Estágio } \\
\text { sucessional }\end{array}$} \\
\hline & $\mathrm{Ht}<5 \mathrm{~m}$ & $5 \mathrm{~m} \leq \mathrm{Ht}<12 \mathrm{~m}$ & $\mathrm{Ht} \geq 12 \mathrm{~m}$ & & EI & EM & EA & \\
\hline 1 & 9 & 51 & 21 & 81 & $11 \%$ & $63 \%$ & $26 \%$ & EM/EA \\
\hline 2 & 28 & 56 & 32 & 116 & $24 \%$ & $48 \%$ & $28 \%$ & EM/EA \\
\hline 3 & 13 & 59 & 68 & 140 & $9 \%$ & $42 \%$ & $49 \%$ & EA \\
\hline 4 & 18 & 70 & 7 & 95 & $19 \%$ & $74 \%$ & $7 \%$ & EM \\
\hline 5 & 28 & 84 & 26 & 138 & $20 \%$ & $61 \%$ & $19 \%$ & EM/EA \\
\hline 6 & 13 & 61 & 32 & 106 & $12 \%$ & $58 \%$ & $30 \%$ & EM/EA \\
\hline 7 & 25 & 48 & 24 & 97 & $26 \%$ & $49 \%$ & $25 \%$ & EM/EA \\
\hline 8 & 9 & 76 & 55 & 140 & $6 \%$ & $54 \%$ & $39 \%$ & EM/EA \\
\hline 9 & 13 & 76 & 11 & 100 & $13 \%$ & $76 \%$ & $11 \%$ & EM/EA \\
\hline 10 & 17 & 91 & 18 & 126 & $13 \%$ & $72 \%$ & $14 \%$ & EM/EA \\
\hline 11 & 12 & 66 & 48 & 126 & $10 \%$ & $52 \%$ & $38 \%$ & EM/EA \\
\hline 12 & 16 & 130 & 22 & 168 & $10 \%$ & $77 \%$ & $13 \%$ & EM/EA \\
\hline 13 & 59 & 88 & 21 & 168 & $35 \%$ & $52 \%$ & $13 \%$ & EM/EA \\
\hline 14 & 34 & 87 & 1 & 122 & $28 \%$ & $71 \%$ & $1 \%$ & EM \\
\hline 15 & 15 & 93 & 19 & 127 & $12 \%$ & $73 \%$ & $15 \%$ & EM/EA \\
\hline 16 & 19 & 91 & 15 & 125 & $15 \%$ & $73 \%$ & $12 \%$ & EM/EA \\
\hline 17 & 24 & 108 & 11 & 143 & $17 \%$ & $76 \%$ & $8 \%$ & EM \\
\hline 18 & 30 & 121 & 8 & 159 & $19 \%$ & $76 \%$ & $5 \%$ & EM \\
\hline 19 & 51 & 117 & 0 & 168 & $30 \%$ & $70 \%$ & $0 \%$ & EM \\
\hline 20 & 49 & 99 & 1 & 149 & $33 \%$ & $66 \%$ & $1 \%$ & EM \\
\hline Média & 24 & 84 & 22 & & $18 \%$ & $64 \%$ & $18 \%$ & EM/EA \\
\hline Total & 482 & 1672 & 440 & 2594 & & & & \\
\hline
\end{tabular}

Fonte: Autores (2020)

Em que: $\mathrm{El}$ = estágio inicial; EM = estágio médio; EA = estágio avançado. 
De acordo com a Tabela 1 e a Figura 2, observa-se que a comunidade, tal como as florestas nativas multiestratificadas (SPURR; BARNES, 1980; GUILHERME; MORELLATO; ASSIS, 2004; NEWTON, 2007; COLONETTI et al., 2009), contêm, além do sub-bosque, três estratos de altura total, o que é típico dos estágios serais ou sucessionais secundários avançados e clímax ou primário, mas como, no caso, é uma área antropizada (TEIXEIRA, 2009), pode indicar um estágio avançado de regeneração da vegetação secundária (BRASIL, 2007).

Como se trata de espécie típica do estrato médio da floresta, o estado de conservação do fragmento reflete a forte presença de Euterpe edulis. Logo, a conservação de fragmentos florestais é de suma importância para a ocorrência da referida espécie, principalmente no que tange à produção de frutos com fins de manejo de produtos não madeireiros, uma vez que áreas perturbadas e com baixa densidade de indivíduos e de polinizadores tendem a ter provável diminuição de produção de frutos.

Figura 2 - Distribuição por classe de altura, sendo a comunidade representada pelo gráfico a (ajuste f.d.p pelo modelo de Hoerl), e a população de Euterpe edulis representada pelo gráfico b (ajuste f.d.p pelo modelo Vapor Pressure)

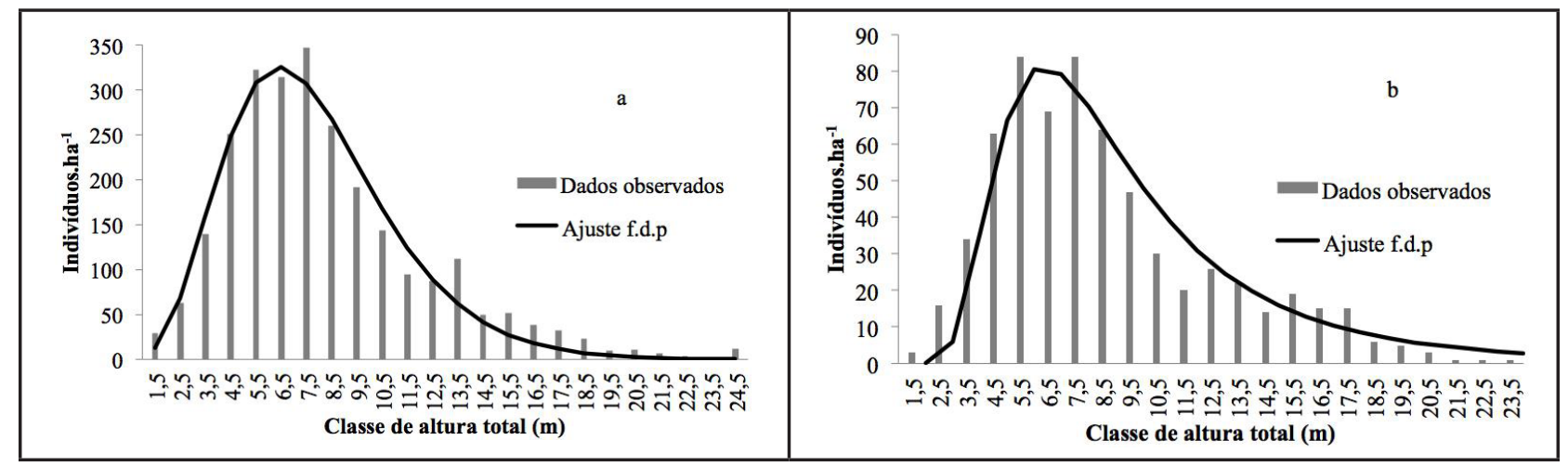

Fonte: Autores (2020)

O modelo de Hoerl $\left(Y_{i}=a b^{x} x^{c}\right)$ foi o que apresentou o melhor ajuste da função densidade probabilidade (f.d.p) para a distribuição de altura (Figura 2a) com amplitude de classe de 1,0 metro (Syx $= \pm 21,15 ; r=0,9838)$, resultando nos seguintes parâmetros: $a=5,8013996 ; b=0,4841823 ; c=4,6692853$. No entanto, considerando 
apenas a população de Euterpe edulis (Figura 2b), o modelo Vapor Pressure $\left(Y_{i}=e^{a+b / x+}\right.$ c.lnx) foi o que melhor ajustou a f.d.p (Syx $= \pm 6,26 ; r=0,9755)$, resultando nos seguintes parâmetros: $a=19,03839 ; b=-31,00448 ; c=-5,28695$

A espécie Euterpe edulis apresentou padrão de distribuição agregado $\left(\mathrm{IM}_{i}=1,34\right.$, significativo a $1 \%$ de probabilidade pelo teste F). O padrão de distribuição agregado, apresentado pela espécie Euterpe edulis, pode estar associado a fatores abióticos, como incidência de luz solar e disponibilidade hídrica no solo, como discutido por Alves (1994), indicando existência de competição intraespecífica devido à agregação dos indivíduos da mesma espécie.

A estrutura diamétrica da comunidade florestal (Figura 3) apresentou distribuição em forma de "J-invertido", porém, com curva desbalanceada, com interrupção na classe $67,5 \mathrm{~cm}$, e apenas um único indivíduo ocupando a última classe $(72,5 \mathrm{~cm})$. Essa distribuição demonstra tendência ao aumento da densidade absoluta, causado pelo recrutamento de indivíduos nas menores classes de diâmetro, com tendência crescente no coeficiente " $\mathrm{q}$ " (De Liocourt).

De acordo com Reis (1995), indivíduos de Euterpe edulis mais desenvolvidos apresentam tendência menos agregada decorrente da competição sofrida em estágios anteriores que segregam indivíduos no espaço.

$\mathrm{Na}$ área de estudo foi possível visualizar esse fenômeno ocorrendo; alta densidade de Euterpe edulis no banco de plântulas, principalmente em áreas de clareiras, e, à medida que a densidade de indivíduos da comunidade aumenta, verificase uma drástica redução na ocorrência da espécie Euterpe edulis. Essa afirmação, no entanto, não foi mensurada, apenas observada empiricamente.

O modelo geométrico $\left(Y_{i}=a x^{b x}\right)$ foi o que apresentou o melhor ajuste da função densidade de probabilidade (f.d.p) para a distribuição diamétrica da comunidade (Figura 3) com amplitude de classe de 1,0 cm (Syx $= \pm 5,03 ; r=0,9980)$, resultando nos seguintes parâmetros: $a=430,66402 ; b=-0,03656$. No entanto, considerando apenas a população de Euterpe edulis (Figura 4a), o modelo polinomial de quarto grau $\left(Y_{i}=\right.$ 
$\left.a+b x+c x^{2}+d x^{3}+e x^{4}\right)$ foi o que melhor ajustou a f.d.p (Syx $\left.= \pm 6,56 ; r=0,9686\right)$, resultando nos seguintes parâmetros: $a=566,10933 ; b=-151,41589 ; c=15,40175 ; d=-0,66094$; $\mathrm{e}=0,01004$.

Figura 3 - Distribuição da classe diamétrica da comunidade com representação do ajuste f.d.p (modelo geométrico) e amplitude de classe 1,0 cm

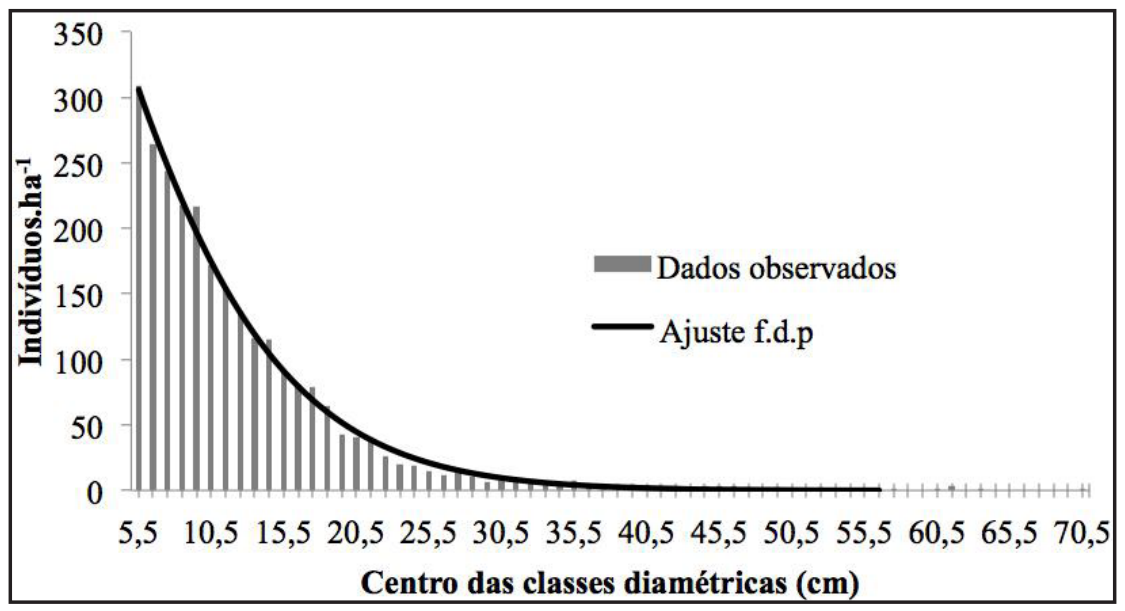

Fonte: Autores (2020)

Como pode ser observada na Figura 4a, a distribuição encontrada para a espécie Euterpe edulis apresenta uma inversão na curva entre as classes de diâmetro 13,5 cm e 17,5 cm. Associando ao resultado encontrado para a distribuição diamétrica dos indivíduos reprodutivos (Figura 4b), e se for considerado que a espécie demora de 7 a 8 anos para atingir a maturidade (REIS, 1995), o resultado da distribuição diamétrica da espécie Euterpe edulis (Figura 4a) pode ser um reflexo da interrupção da exploração clandestina de palmito na área de estudo há mais ou menos 7 anos. Ou seja, devido à redução do desmatamento e do abate de indivíduos de Euterpe edulis para exploração do palmito na área de estudo, em um período marcado pela minimização do impacto social decorrente do êxodo rural, sugere-se que o aumento da densidade absoluta nessa classe de diâmetro implica o aumento da taxa de recrutamento de uma nova geração de indivíduos que sobreviveram em um ambiente com menor pressão antrópica. Analisando a distribuição diamétrica das palmeiras produtivas (Figura 4b), 
que tende à normalidade, pode-se inferir sobre a maturidade da população de Euterpe edulis. De acordo com Reis (1995), é razoável admitir que uma distribuição próxima à normal possa ser encontrada para indivíduos adultos em populações maduras.

Figura 4 - Distribuição diamétrica da população de Euterpe edulis representada pelo gráfico a (ajuste f.d.p pelo modelo polinômio de $4^{\circ}$ grau), e dos indivíduos de Euterpe edulis que apresentaram alguma fenofase durante a coleta dos dados representada pelo gráfico b (ajuste f.d.p pelo modelo Gaussiano)

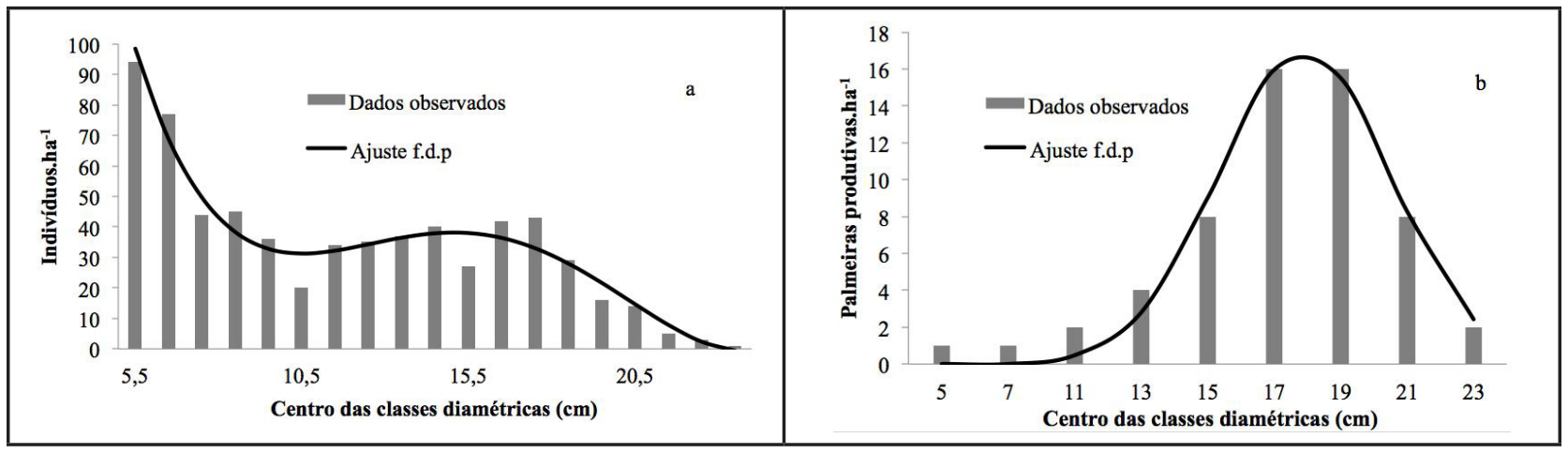

Fonte: Autores (2020)

No caso específico das palmeiras produtivas (Figura 4b), o modelo Gaussiano ( $Y_{i}$ $\left.=a \cdot \exp \left(-(x-b)^{2} / 2 c^{2}\right)\right)$ foi o que melhor ajustou a f.d.p $(\mathrm{Syx}= \pm 1,10 ; r=0,9874)$, resultando nos seguintes parâmetros: $a=16,96619 ; b=17,90627 ; c=2,58273$.

Quanto à estrutura interna da comunidade, houve diferença significativa entre a presença e ausência de epífitas, trepadeiras e cipós. As epífitas, compreendidas por líquens, briófitas, pteridófitas, orquídeas, bromélias e cactos, foram observadas na maioria dos indivíduos amostrados (96,42\%). A abundância de epífitas na comunidade (96,42\%) sugere estágio avançado de sucessão, de acordo com a Resolução Conama n. 392 (BRASIL, 2007), confirmado pela estimativa de pelo menos um indivíduo com presença de orquídea, bromélia ou cacto a cada cinco indivíduos observados $(20,19 \%)$. Essa abundância também pode estar associada ao gradiente altitudinal da área de estudo (1.100 a $1.380 \mathrm{~m}$ ), uma vez que altitudes mais elevadas favorecem a presença de musgos, líquens e epífitas vasculares nas árvores (DOUMENGE et al., 1995 apud 
SAPORETTI JÚNIOR, 2005). Outra associação à abundância de epífitas é feita por Klein (1980), que destaca a importância fisionômica das epífitas (Bromeliaceae, Cactaceae e Orchidaceae) e das lianas (Araceae) em formações de Floresta Ombrófila Densa.

As trepadeiras, ou lianas, foram encontradas em quase metade dos indivíduos (42,21\%), com predominância de herbáceas (22,69\%). O fato de as trepadeiras, ou lianas, terem sido encontradas em quase metade dos indivíduos, com predominância de herbáceas, pode ser mais um quesito para interpretação do estágio sucessional, sugerindo, neste caso, que a comunidade se encontra em estágio médio de sucessão, segundo Resolução Conama n. 392 (BRASIL, 2007). Cipós foram observados em 37,5\% dos indivíduos, sendo que a maioria está presente somente no tronco (20,31\%). De acordo com a Resolução Conama n. 392 (BRASIL, 2007), o estágio avançado de sucessão apresenta menor densidade de cipós e arbustos em relação ao estágio médio, em que a presença de cipós é marcante. Nesse sentido, considera-se que a comunidade assemelha-se mais ao estágio médio de sucessão, contudo houve diferença significativa entre a presença e ausência de cipós.

A estratificação vertical da frequência das espécies indicou forte presença no estrato superior com média de 18\%, mas chegando a 49\% na parcela 3, sendo possível afirmar que a comunidade está em transição para o estágio avançado. Essa situação foi observada em mais da metade das parcelas e a resolução indica a necessidade de predominância dessa característica para proceder a classificação.

Do total de 642 indivíduos de Euterpe edulis por hectare, 349 indivíduos.ha-1 $^{-1}$ coabitam ambientes sombreados e, destes, somente quatro estavam em fenofase reprodutiva, ao passo que 35 indivíduos produtivos.ha-1 coabitavam o ambiente na condição de iluminação total de copa e 19 sob iluminação parcial. O predomínio de indivíduos produtivos de Euterpe edulis sob condição de iluminação total, contrastando com a maioria dos indivíduos da espécie coabitando ambiente sombreado, sugere que os níveis de luminosidade exigidos pela espécie Euterpe edulis variam de acordo com o estágio ontogenético em que se encontra (PORTELA; SANTOS, 2011). 
De acordo com a variável qualidade de copa, não foram observadas palmeiras produtivas para o estrato inferior, sendo a grande maioria das palmeiras produtivas $(93,22 \%)$ observada para condição de copa boa.

\section{CONCLUSÃO}

O fragmento encontra-se em estágio de transição do médio para avançado de regeneração da Mata Atlântica, contudo o estrato vertical superior apresenta destaque com média de 18\% de frequência das espécies e 65\% das parcelas foram classificadas como sendo transição de estágios de sucessão, assim como parâmetros avaliados de forma qualitativa como abundância de epífitas, sub-bosque e presença de serapilheira, evidências que corroboram a presente classificação.

A densidade populacional de Euterpe edulis encontrada é condizente com estudos populacionais da espécie. Porém, a estimativa dos indivíduos adultos está subestimada, uma vez que não foram considerados os demais sinais de evidência reprodutiva. Ainda assim, a representação da espécie pela área basal demonstra a sua dominância na comunidade florestal em que se encontra, indicando o grau de maturidade da população estudada.

O padrão de distribuição agregado da espécie Euterpe edulis pode favorecer sua coleta para práticas de cultivo, integrando lavoura, pomares ou quintais e recuperação de áreas de preservação permanente, sobretudo matas ciliares e grotas de nascentes, uma vez que a competição intraespecífica pode ser uma característica ecológica da espécie.

\section{AGRADECIMENTOS}

À Universidade Federal de Viçosa e ao Departamento de Engenharia Florestal pela oportunidade de realizar o mestrado em Ciências Florestais e ao Conselho Nacional de Desenvolvimento Científico e Tecnológico pela concessão da bolsa de estudos. 


\section{REFERÊNCIAS}

ÁLVARES, C. A. et al. Köppen`s climate classification map for Brazil. Meteorologische Zeitschrift, Sttutgart, v. 22, n. 6, p. 711-728, 2013.

ALVES, L. F. Competição Intraespecífica e padrão espacial em uma população de $E$. edulis Mart. (Arecaceae). 1994. Dissertação (Mestrado em Biologia Vegetal) - Instituto de Biologia, Universidade Estadual de Campinas, Campinas, 1994.

BORÉM, R. A. T.; OLIVEIRA-FILHO, A. T. Fitossociologia do Estrato Arbóreo em uma Topossequência Alterada de Mata Atlântica, no Município de Silva Jardim-RJ, Brasil. Revista Árvore, Viçosa, MG, v. 26, n. 6, p. 727-742, 2002.

BRASIL. Ministério do Meio Ambiente. Portaria MMA n 443, de 17 de dezembro de 2014. Brasília, 2014. Disponível em: http://www.ibama.gov.br. Acesso em: 12 set. 2017.

BRASIL. Ministério do Meio Ambiente. Resolução Conama Nº 392, de 25 de junho de 2007. Definição de vegetação primária e secundária de regeneração de Mata Atlântica no Estado de Minas Gerais. Brasília, 2007. Disponível em: http://www.mma.gov.br. Acesso em: 10 ago. 2009.

BROWER, J. R.; ZAR, J. H. Field \& laboratory methods for general ecology. Dubuque: W. C. Brown Publishers, 1984. 226 p.

CAMPOS, E. P. et al. Florística e Estrutura Horizontal da Vegetação Arbórea de uma Ravina em um Fragmento Florestal no Município de Viçosa, MG. Revista Árvore, Viçosa, MG, v. 30, n. 6, p. 1045-1054, 2006.

CARVALHO, P. E. R. Espécies florestais brasileiras: recomendações silviculturais, potencialidades e uso da madeira. Brasília, DF: EMBRAPA; SPI, 1994. 639 p.

CARVALHO, P. E. R. Espécies Arbóreas Brasileiras. Colombo, PR: Embrapa Florestas, 2003. $1039 \mathrm{p}$.

COLONETTI, S. et al. Florística e estrutura fitossociológica em floresta ombrófila densa submontana na barragem do rio São Bento, Siderópolis, Estado de Santa Catarina. Acta Scientiarum. Biological Sciences, Maringá, v. 31, n. 4, p. 397-405, 2009.

ENGEVIX S. A. Caracterização do meio físico da área autorizada para criação do Parque Estadual da Serra do Brigadeiro-relatório técnico final dos estudos.8292-RG-H4-003/94, "VER 1". [S. I.]: Instituto Estadual de Floresta; Bird; Prófloresta; Seplan, 1995. 34 p.

FOOD AND AGRICULTURE ORGANIZATION OF THE UNITED NATIONS. Forest volume estimation and yield prediction: yield prediction by $d$. Alder commonwealth forestry institute, u. k. Rome, 1980. v. 2. 20 p.

FUNDAÇÃO SOS MATA ATLÂNTICA; INSTITUTO NACIONAL DE PESQUISAS ESPACIAIS (Brasil). Atlas dos remanescentes Florestais da Mata Atlântica período 2013-2014: relatório técnico. São Paulo, 2015.

GOMES, F. P. A estatística moderna na pesquisa agropecuária. 3. ed. Piracicaba: Potafós, 1987. 162 p. 
GUILHERME, F. A. G.; MORELLATO, P. C.; ASSIS, M. A. Horizontal and vertical tree community structure in a lowland Atlantic Rain Forest, Southeastern Brazil. Revista Brasileira de Botânica, São Paulo, v. 27, n. 4, p. 725-737, 2004.

HIGASHIKAWA, E. M. Fitossociologia de um fragmento florestal como monodominância de Euterpe edulis Mart. 2009. Dissertação (Mestrado em Engenharia Florestal) - Universidade Federal de Lavras, Lavras, 2009.

HIGUCHI, P. et al. Composição Florística da Regeneração Natural de Espécies Arbóreas ao Longo de Oito Anos em um Fragmento de Floresta Estacional Semidecidual, em Viçosa, MG. Revista Árvore, Viçosa, MG, v. 30, n. 6, p. 893-904, 2006.

IBGE. Manual técnico da vegetação brasileira. Rio de Janeiro, 2012. 271 p. (Manuais técnicos em geociências, n. 1).

INSTITUTO ESTAdUAL DE FlORESTAS (MG). Plano de Manejo do Parque Estadual Serra do Brigadeiro, Minas Gerais. [S. I.], 2008.

IRSIGLER, D. T. Composição florística e estrutura de um trecho primitivo de floresta estacional semidecidual em Viçosa, MG. 2002. Dissertação (Mestrado em Botânica) - Centro de Ciências Biológicas, Universidade Federal de Viçosa, Viçosa, MG, 2002.

KLEIN, R. M. Ecologia da flora e vegetação do Vale do Itajaí. Sellowia, Itajaí, v. 32, p. 164-369, 1980.

KREBS, C. J. Ecological methodology. 2th ed. New York: Benjamin; Cummings, 1999. 581 p.

LEITE, H. G.; OLIVEIRA, F. H. T. Statistical procedure to test the identity of analytical methods. Communications in Soil Science and Plant Analysis, New York, v. 33, p. 1105-1118, 2002.

LEMOS, P. H. D. Efeito de borda no componente arbóreo de um fragmento de floresta semidecídua, Viçosa, MG. 2008. Dissertação (Mestrado em Botânica) - Centro de Ciências Biológicas, Universidade Federal de Viçosa, Viçosa, MG, 2008.

LIMA, M. E. L.; CORDEIRO, I.; MORENO, P. R. H. Estrutura do Componente Arbóreo em Floresta Ombrófila Densa Montana no Parque Natural Municipal Nascentes de Paranapiacaba (PNMNP), Santo André, SP, Brasil. Hoehnea, São Paulo, v. 38, n. 1, p. 73-96, 2011.

LORENZON, A. S. Processos Hidrológicos em um Fragmento de Floresta Estacional Semidecidual no Município de Viçosa, MG. 2011. Dissertação (Mestrado em Ciência Florestal) - Universidade Federal de Viçosa, Viçosa, MG, 2011.

MACHADO, S. A. et al. Distribution of total height, transverse area and individual volume for Araucaria angustifolia (Bert.) O. Kuntze. Revista Cerne, Lavras, v. 16, n. 1, p. 12-21, 2010.

MEYER, H. A. Structure, growth and drain in balanced uneven-aged forests. JournalofForestry, Washington, v. 50, p. 85-92, 1952.

MOREIRA, L. S. Socioecologia de muriquis-do-norte (Brachyteles hypoxanthus) no Parque Estadual da Serra do Brigadeiro, MG. 2008. Dissertação (Mestrado em Biologia Animal) Universidade Federal de Viçosa, Viçosa, MG, 2008. 
MORENO, M. R.; NASCIMENTO, M. T.; KURTZ, B. C. Estrutura e Composição Florística do Estrato Arbóreo em duas Zonas Altitudinais na Mata Atlântica de Encosta da Região de Imbé, RJ. Acta Botânica Brasileira, São Paulo,v. 17, n. 3, p. 371-386, 2003.

NEWTON, A. C. Forest ecology and conservation: a handbook of techniques. New York: Oxford University Press, 2007. 454 p.

NODARI, R. O. et al. Restauração de populações de E. edulis Martius (Arecaceae) na Mata Atlântica. Sellowia, Itajaí, v. 49/52, p. 189-201, 2000.

OLIVEIRA-FILHO, A. T. Catálogo das árvores nativas de Minas Gerais: mapeamento e inventário da flora nativa e dos reflorestamentos de Minas Gerais. Lavras: Editora UFLA, 2006. $423 \mathrm{p}$.

PINTO, S. I. C. et al. Estrutura do Componente Arbustivo-arbóreo de dois Estádios Sucessionais de Floreta Estacional Semidecidual na Reserva Florestal Mata do Paraíso, Viçosa, MG, Brasil. Revista Árvore, Viçosa, MG, v. 31, n. 5, p. 823-833, 2007.

PORTELA, R. C. Q.; SANTOS, F. A. M. Caracterização dos estádios ontogenéticos de três espécies de palmeiras: uma proposta de padronização estudos de dinâmica populacional. Revista Brasileira de Botânica, São Paulo, v. 34, n. 4, p. 523-535, 2011.

PRATA, E. M. B.; ASSIS, M. A.; JOLY, C. A. Composição Florística e Estrutura da Comunidade Arbórea na Transição da Floresta Ombrófila Densa das Terras Baixas - Floresta Ombrófila Densa Submontana do Núcleo Picinguaba/PESM, Ubatuba, Sudeste do Brasil. Biota Neotropica, Campinas,v. 11, n. 2, 2011.

RAMOS, A. M.; SANTOS, L. A. R. D; FORTES, L. T. G. Normais climatológicas do Brasil 1961-1990:edição revista e ampliada. Brasília: INMET, 2009.

REIS, A. Dispersão de Sementes de E. edulis Martius - (Palmae) em uma Floresta Ombrófila Densa Montana da Encosta Atlântica em Blumenau, SC. 1995. Tese (Doutorado em Biologia Vegetal) - Instituto de Biologia, Universidade Estadual de Campinas, Campinas, 1995.

REITZ, R. Palmeiras. Itajaí: Herbário Barbosa Rodrigues, 1974. 189 p.(Flora llustrada Catarinense - PALM).

RIBEIRO, C. A. N. Florística e Fitossociologia de um Trecho de Floresta Atlântica de Altitude na Fazenda da Neblina, Parque Estadual da Serra do Brigadeiro, Minas Gerais.2003. Dissertação (Mestrado em Botânica) - Universidade Federal de Viçosa, Viçosa, MG, 2003.

RIBEIRO, C. S. et al. Quantificação de biomassa e estimativa de estoque de carbono em uma floresta madura no município de Viçosa, Minas Gerais. Revista Árvore, Viçosa, MG, v. 23, n. 5, p. 917-926, 2009a.

RIBEIRO, M. C. et al. The Brazilian Altantic Forest: how much is left, and how is the remaining forest distributed? Implications for conservation. Biological Conservation, Amsterdam, v. 142, p. 1141-1153, 2009b. 
SÁ JÚNIOR, A. et al. Application of the Köppen classification for climatic zoning in the state of Minas Gerais, Brazil. Theoretical Applied Climatology, Wien, v. 108, p. 1-7, 2012.

SAPORETTI JÚNIOR, A. W. Composição Florística e Estrutura do Componente Arbóreo em um Remanescente de Floresta Atlântica Montana, Araponga, MG. 2005. Dissertação (Mestrado em Botânica) - Universidade Federal de Viçosa, Viçosa, MG, 2005.

SILVA JÚNIOR, W. M. Ecologia de Florestas Atlânticas com Ocorrência do Muriqui (Brachyteles spp.): diversidade, sucessão secundária e estrutura nutricional. 2008. Tese (Doutorado em Botânica) - Centro de Ciências Biológicas, Universidade Federal de Viçosa, Viçosa, MG, 2008.

SILVA, M. G. C. P. C. et al. Estrutura populacional de E. edulis Mart. no Sul da Bahia. Revista Brasileira de Botânica, São Paulo, v. 32, n. 2, p. 393-403, 2009.

SILVA, S. S. Estrutura e Dinâmica de Populações de Espécies Arbóreas em uma Floresta Estacional Semidecidual Aluvial em Viçosa - MG.2003. Dissertação (Mestrado em Botânica) - Universidade Federal de Viçosa, Viçosa, MG, 2003.

SPURR, S. H.; BARNES, B. V. Forest Ecology. 3th ed. New York: John Wiley \& Sons, 687. p. 1980.

TABARELI, M. et al. Challenges and opportunities for biodiversity conservation in the Brazilian Atlantic forest. Conservation Biology, New Jersey, v. 19, p. 695-700, 2005.

TEIXEIRA, A. P.; ASSIS, M. A. Caracterização Florística e Fitossociológica do Componente Arbustivo-Arbóreo de uma Floresta Paludosa no Município de Rio Claro (SP), Brasil. Revista Brasileira de Botânica, São Paulo, v. 28, n. 3, p. 467-476, 2005.

TEIXEIRA, M. C. C. Reaprendendo a conviver com os macacos na Serra do Brigadeiro. 2009. Dissertação (Doutorado em Ciências Sociais em Desenvolvimento, Agricultura e Sociedade) Universidade Federal Rural do Rio de Janeiro, Soropédica, RJ, 2009.

VELOSO, H. P.; KLEIN, R. M. As comunidades e associações vegetais da mata pluvial atlântica do sul do Brasil. I. As comunidades do Município de Brusque. Estado de Santa Catarina. Sellowia, Itajaí, v. 8, p. 81-235, 1957.

VELOSO, H. P.; RANGEL FILHO, A. L. R.; LIMA, J. C. Classificação da vegetação brasileira, adaptada a um sistema universal. Rio de Janeiro: IBGE, 1991. 125 p. 


\section{Contribuição de Autoria}

\section{1 - Letícia Bonifácio Fávaro}

Engenheira Florestal, Me., Pesquisadora Autônoma

https://orcid.org/0000-0002-7569-0793•leticia.bonifacio@gmail.com

Contribuição: Conceituação, Curadoria de dados, Análise Formal, Obtenção de financiamento, Investigação, Administração do projeto, Supervisão, Validação, Visualização de dados, Escrita - primeira redação, Escrita - revisão e edição

\section{2 - Agostinho Lopes de Souza}

Engenheiro Florestal, Dr., Professor

https://orcid.org/0000-0003-0205-2392 • alsousaal@gmail.com

Contribuição: Conceituação, Análise Formal, Investigação, Metodologia, Recursos, Validação, Visualização de dados, Escrita - primeira redação, Escrita - revisão e edição

\section{3 - Renato Totti Maia}

Engenheiro Ambiental

https://orcid.org/0000-0001-7135-5579•renato_maia@ima.sc.gov.br

Contribuição: Curadoria de dados, Investigação

\section{4 - Ricardo de Oliveira Gaspar}

Engenheiro Florestal, Dr., Professor

https://orcid.org/0000-0002-2035-2180•ricogaspar@unb.br

Contribuição: Análise Formal, Validação, Visualização de dados, Escrita - revisão e edição

\section{Como citar este artigo}

Fávaro, L. B.; Souza, A. L.; Maia, R. T.; Gaspar, R. O. Estrutura de uma Floresta Ombrófila Densa Montana com dominância de Euterpe edulis Mart. Ciência Florestal, Santa Maria, v. 31, n. 2, p. 550-568, 2021. DOI 10.5902/1980509810699. Disponível em: https://doi. org/10.5902/1980509810699. Acesso em: xx mês-abreviado 2021. 